\title{
An all-sky set of $(B)-V-R$ photometric calibrators for Schmidt surveys
}

\section{GSPC2.1: First release}

\author{
B. Bucciarelli ${ }^{1}$, J. García Yus ${ }^{2,3,4}$, R. Casalegno ${ }^{1}$, M. Postman ${ }^{2,4}$, B. M. Lasker ${ }^{2}$, C. Sturch ${ }^{2,5}$,
} M. G. Lattanzi ${ }^{1}$, B. J. McLean ${ }^{2}$, E. Costa ${ }^{6,7}$, A. Falasca ${ }^{2}$, R. Le Poole ${ }^{8}$, G. Massone ${ }^{1}$, M. Potter $^{2,4}$, A. Rosenberg ${ }^{2,4}$, T. Borgman ${ }^{4}$, J. Doggett ${ }^{2,4}$, J. Morrison ${ }^{2,4}$, A. Pizzuti ${ }^{4}$, E. Pompei ${ }^{9}$, D. Rehner $^{4}$, L. Siciliano ${ }^{7}$, and D. Wolfe ${ }^{2,4}$

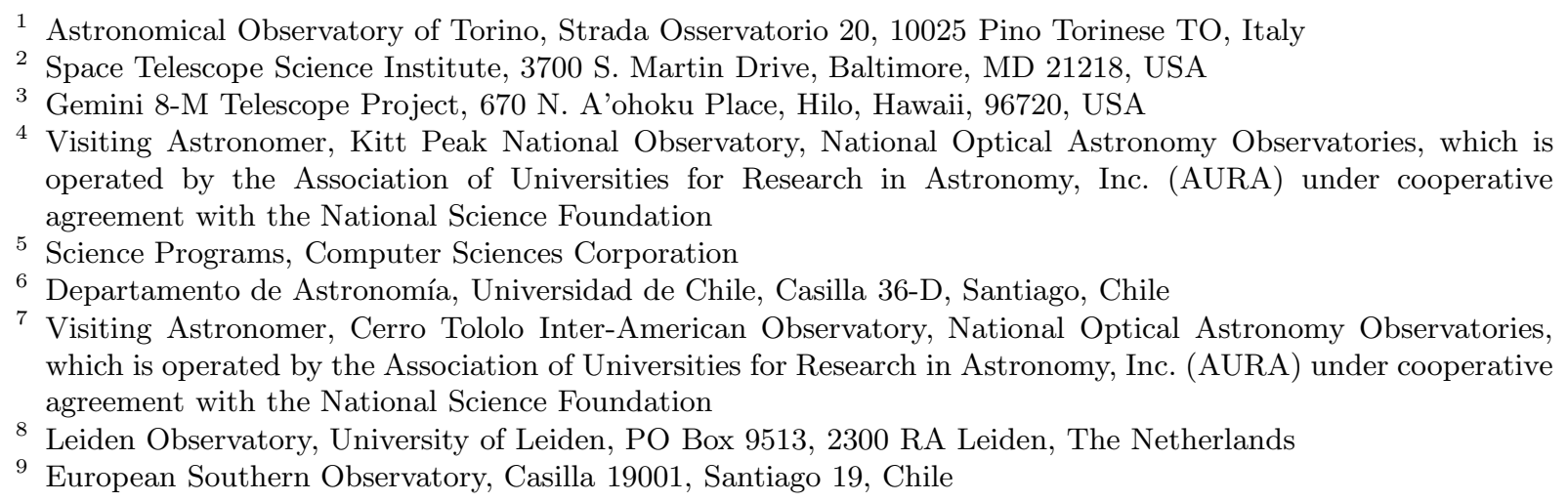

Received 31 October 2000 / Accepted 5 December 2000

\begin{abstract}
We publish 622 CCD photometric sequences in the Johnson-Kron-Cousins standard system, distributed both in the northern and southern hemispheres, useful for the calibration of photographic photometry of Schmidt survey plates. The collection and reduction of the CCD data presented here are part of a long-term program devoted to the construction of the Second Guide Star Photometric Catalog (GSPC-II). The GSPC-II is an all-sky catalog of photometric stellar sequences with a limiting magnitude of $V=19$ or fainter, in the $(B), V$, and $R$ passbands of the Johnson-Kron-Cousins system. Standard photometric errors are at the level of $\sim 0.07$ for a $V \sim 19$ magnitude star. These sequences are being used by teams of the Space Telescope Science Institute (STScI) and the Osservatorio Astronomico of Torino (OATo) for the photometric calibration of the Second Guide Star Catalog.
\end{abstract}

Key words. instrumentation: detectors - methods: miscellaneous - techniques: photometric - catalogs - surveys

\section{Introduction}

The last decade has witnessed a multiplication of efforts towards proper exploitation of all-sky Schmidt surveys. The principal requirement to this end is the use of fast measuring machines for the storage of the full astronomical information present in each plate, equivalent to about half a billion pixels. Extraction of the final astronomical parameters, i.e., single object classification, astrometry, and photometry, entails the development of ad-hoc

\footnotetext{
Send offprint requests to: B. Bucciarelli

* Based on observations collected at the European Southern Observatory, Chile.
}

reduction algorithms, which must not prescind from the digitization process. Various programs have been undertaken in this direction, including the construction of the second generation Guide Star Catalog (GSC-II) initially promoted by the STScI and later by a multi-partner collaboration ${ }^{1}$. The original motivations for such an endeavour were driven by the required enhancements of the first

\footnotetext{
1 The GSC-II project is a joint effort of STScI and OATo, with support by the Italian Council for Research in Astronomy, the International GEMINI project, the European Southern Observatory and Space Telescope European Coordinating Facility, and the European Space Agency Astrophysics Division.
} 
Guide Star Catalog (GSC, Lasker et al. 1990) to continue supporting the HST mission. Currently the GSC partially meets HST specifications to provide guide stars with relative positions to about $0.3^{\prime \prime}$ and photometry to better than 0.4 mag for telescope pointing and observation planning. Because it is a single epoch catalog, however, the errors due to proper motions presently exceed $1^{\prime \prime}$ in some parts of the sky. Additionally, it is important to have at least one color to $V \simeq 18$ for planning purposes, such as verifying the absence of bright objects in camera fields. Similarly, new ground- and space-based telescopes will require access to a catalog of faint guide stars with comparable astrometry and photometry to provide data for active optics systems, target acquisition, observation planning, and efficient scheduling. These needs will be fulfilled by the GSC-II, with proper motions and colors to at least $V=18$ based on multi-color and multi-epoch Schmidt surveys (Lasker et al. 1995; Bucciarelli 1999; McLean et al. 2000).

Besides its operational use, the very nature of GSC-II makes it a tremendous resource to address a large variety of astrophysical investigations. In particular, GSC-II data can be successfully exploited for studies of Galactic structure and kinematics, where accurate astrometry and photometry are essential requirements (see, e.g., Spagna et al. 1996; Spagna et al. 1998; Terranegra et al. 1999; Sciortino et al. 2000).

Ultimately, the availability of external reference objects is of utmost importance for the calibration of the intrinsically non-linear response of photographic data. In particular, to derive good stellar photometry out of the survey plates one needs reference stars over the whole sky, covering a large dynamic range in brightness in at least two passbands.

About ten years ago, common scientific interests in the field of Schmidt astronomy led the STScI and the OATo to the definition of a joint program for the collection of an all-sky set of deep, multi-color photometric sequences. This program has produced a database with a wealth of CCD stellar sequences currently employed to calibrate the STScI Schmidt plate archive. The purpose of these sequences, in their present form, is restricted to the calibration of photographic plates, as opposed to providing accurate CCD photometry of single objects, which will be the goal of future releases of GSPC-II.

The aim of this paper is twofold: (a) give a detailed account of the observing program carried out by STScI and OATo, as well as of the content and methods of contruction of the current CCD data archive; (b) make available to the astronomical community an all-sky set of CCD photometric calibrators. The photometric quality of the sequences presented here is assessed by global statistics based on the fits to the Landolt's standard stars and on direct comparison against the GSPC-I catalog (Lasker \& Sturch et al. 1988), as well as by semi-external checks of single object photometry in selected fields.

\section{The GSPC-II program}

The bulk of the GSPC-II target fields coincides with those of the GSPC-I created to provide photometric calibrators for the first Guide Star Catalog. The GSPC-I consists of 1477 photoelectric sequences, with distribution determined by the survey plates used in the GSC. Specifically, there are 580 northern $\left(\delta \geq 6^{\circ}\right)$ sequences $^{2}$, centered on the $6^{\circ}$-grid (equinox 1855) of the original National Geographic Society - Palomar Observatory Sky Survey (POSS-I, Minkowski \& Abell 1963) and the "Quick V" survey (Lasker et al. 1990).

There are 894 sequences at $\delta \leq 0^{\circ}$, corresponding to the fields of the SERC Survey and the Equatorial Extension thereto (Cannon 1984), taken on the standard $5^{\circ}$ grid of equinox 1950 coordinates. Five additional sequences were added to GSPC-I after its publication, for better coverage of fields N586, N594, N608, N611 and N613 at $\delta=+3^{\circ}$.

The northern program of the second generation Schmidt surveys includes all 894 fields of the POSS-II (Reid et al. 1991) in three bands: green (IIIa-J), red (IIIa$\mathrm{F})$, and near-IR (IV-N). The southern program consists of the Second Epoch Survey (AAO-R) and the equatorial counterpart, the SERC-ER, both (IIIa-F) surveys (Morgan et al. 1992).

While the AAO-R lies on the same grid pattern as the SERC and therefore does not require additional sequences, the POSS-II survey is placed on a $5^{\circ}$ grid of equinox 1950 coordinates. This results in a number of POSS-II plates being considerably shifted with respect to the original grid, and therefore poorly covered by the GSPC-I sequences. Enforcing the requirement that each photometric sequence lie within $2.7^{\circ}$ from the plate center, in order to avoid the area where the sensitivity of the plate is badly affected by vignetting, adds another 302 sequences to the list. All these additional fields bring the number of GSPCII standard sequences to a total of 1780 .

\subsection{Observations}

All of the GSPC-II data collected to date come from 10 different telescopes, for a total of 337 useful nights. Most of the northern CCD sequences were imaged at the Kitt Peak and Wise Observatories, whereas southern declinations have been mainly covered at the Cerro Tololo and ESO-La Silla observatories. Table 1 gives an up-todate full account of all the telescopes employed, together with the epoch range in which GSPC-II observations were made. Figure 1 displays the position of the centers of all the observed sequences on the celestial sphere in equatorial coordinates. Although a considerable portion of northern data have been collected by the Wise Observatory (Ein Yahav, Israel), the reduction process revealed some deficiencies intrinsic to these data, particularly poor standard

${ }^{2}$ Four northern sequences are shared between pairs of POSSI fields. 


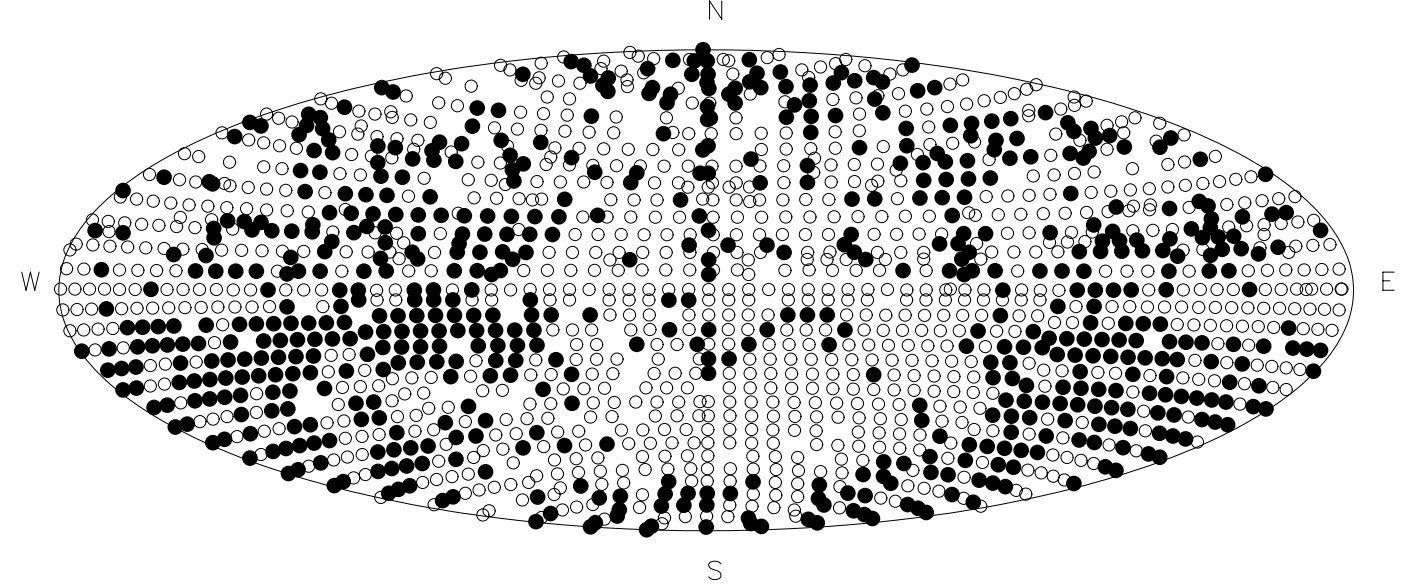

Fig. 1. Distribution of the GSPC-II fields on the equatorial celestial sphere. Open circles refer to the sequences released with this paper, filled circles to the rest of the observed fields. $\mathrm{RA}=0 \mathrm{~h}$ is to the left-hand side

Table 1. Telescope usage and number of GSPC-II sequences collected (as of December 2000)

\begin{tabular}{|c|c|c|c|c|c|c|c|c|}
\hline Telescope & Latitude & Size & \multicolumn{2}{|c|}{ \# of Observed Fields } & Total & Useful & Epoch \\
& $\left(\delta^{\circ}\right)$ & (meters) & POSS-I & POSS-II & SERC-J & Runs & Nights & range \\
\hline Kitt Peak & +31.9 & 0.9 & 323 & 146 & 34 & 17 & 63 & $1990-1999$ \\
Cerro Tololo & -30.0 & 0.9 & 177 & 13 & 488 & 21 & 111 & $1989-1998$ \\
ESO Dutch & -29.2 & 0.9 & - & 6 & 528 & 14 & 103 & $1991-1998$ \\
Wise & +30.6 & 1 & 226 & - & 2 & 24 & 36 & $1989-1991$ \\
Mt. Hopkins & +31.6 & 1.2 & 50 & - & 2 & 4 & 5 & $1994-1995$ \\
Mt. Laguna & +32.8 & 1 & 84 & - & 5 & 2 & 9 & $1990-1999$ \\
Lowell & +35.2 & 1 & 10 & - & - & 1 & 2 & 1990 \\
Mt. Megantic & +45.4 & 1.6 & 6 & - & - & 3 & 1 & 1990 \\
McDonald & +30.6 & 0.8 & 2 & - & 1 & 1 & 1 & 1990 \\
JKT La Palma & +28.7 & 1 & 36 & 16 & 17 & 2 & 6 & $1998-1999$ \\
\hline
\end{tabular}

stars statistics, insufficient number of stars per sequence, absence of good sets of bias and flat field frames. For this reason, re-observations of all of the Wise sequences have been planned, with a current completion percentage of $\sim 88 \%$. Globally, about $50 \%$ of the GSPC-II fields has been observed from different telescopes/multiple nights, allowing a better control of their photometric quality. Finally, about $10 \%$ of the fields remains still uncovered, either because they lack observations or because the existing data were rejected as non photometric.

Given the long time span of this survey project, many detectors have been replaced with new ones over the years. It would be too cumbersome to list here the characteristics of all the CCD cameras available at each telescope at the time each observing run occurred; it suffices to say that the size of the camera field was typically $3.5 \times 3.5$ arcmin for sequences at declinations $<-30^{\circ}, \sim 10$ arcmin for $-30^{\circ}<\delta<0^{\circ}$ and a combination of $\sim 10$ and $\sim 23$ arcmin for the northern hemisphere. As for image sampling, the pixel scale of the different CCDs ranged from $\sim 0.35^{\prime \prime} / \mathrm{px}$ to $\sim 0.7^{\prime \prime} / \mathrm{px}$.

The original goal of the GSPC-II was to extend the photometry in the $V$ and $R$ bands to a limiting magnitude of $V \simeq 18$ with a precision of 0.05 magnitudes, to meet the
HST mission requirements. Where resources were available, which is for a large part of the southern hemisphere and for a few northern fields, a $B$ band was also included, and the limiting magnitude brought to $\simeq 20$. The observation strategy consisted in taking CCD frames centered on the faintest star of the corresponding GSPC-I sequence, where the latter was available. When necessary, both long ( 8-15 $\mathrm{min})$ and short (<3 min) exposures were acquired in each field. Short exposures were chosen to be appropriate for the typical $14.5 \mathrm{mag}$ target star. This serves the purpose of bridging the gap between the bright and faint end of the sequence and, at the same time, enables confirmation of the GSPC-I magnitude. For fields which did not have a GSPC-I counterpart, i.e., those corresponding to the POSS-II grid, the telescope was pointed to the nominal center of the corresponding survey plate, and the pointing adjusted in order to avoid bright stars.

Besides target fields, a pre-selected set of standard stars from the Landolt catalog (Landolt 1992) was regularly imaged through the night to estimate the extinction and the transformation to the standard photometric system, as explained in the following sections. Similarly, several dome, sky flats and bias frames were taken each night to accurately calibrate the instrumental response. 


\section{Data reduction}

Standard photometry has been derived from the CCD images by using ARLO, an ad-hoc pipeline software developed under IRAF 2.10.4 (Casalegno 1998). A detailed description of the pipeline tasks, along with a discussion of the choice of critical input parameters is given in Casalegno et al. (1999). The implementation of the pipeline procedures was driven by two main requirements: the first, building an automated pipeline and the second, storing all the relevant data to perform an error analysis without necessarily going back to the original CCD frames. This is justified by the large amount of data involved, which consists of approximately 30000 images, taken with different telescopes/instruments and over a time span of more than ten years. Both aperture and $P S F$ photometry were routinely performed on each CCD image.

Aperture photometry is computed by the IRAF package $A P P H O T$, with the aperture radius chosen to be equal to 2.3 times the average full-width-half-maximum of the frame, as estimated by the previous IRAF tasks. This choice is driven by the competing requirements of minimizing light contamination from close objects and losing a negligible fraction of the seeing-dominated PSF profile. It turns out that the fraction of light lost with this choice of the radius is less than $1 \%$. In no case is the aperture radius smaller than $4.5^{\prime \prime}$, as suggested in Massey et al. (1989). This circle is supposed to encompass the seeingindependent part of the light distributed in the profile wings.

The fitting method implemented in IRAF is that of DAOPHOT (Stetson 1987). It uses a model for the PSF function made of an analytical part plus an empirical correction table, which is then fit via a least-square technique to derive the best stellar parameters. The choice of the stars being used for the definition of the average PSF profile is an automated procedure not requiring human interaction, as implemented in the ARLO pipeline. This feature has been demonstrated to work well for moderately crowded sky regions, whereas in heavily dense fields a more reliable PSF can be obtained by selecting the stars manually within the frame.

\subsection{Instrumental magnitude errors}

Both instrumental magnitude errors coming from the aperture and PSF methods are carried along during the reductions. The aperture photometry error, as computed by the PHOTCALC task, is given by $\sigma_{\text {ap }}=C / S$. $\sqrt{S / G+A \cdot \sigma_{\mathrm{B}}^{2}+A^{2} \cdot \sigma_{\mathrm{B}}^{2} / N}$, where $C=2.5 \cdot \log (e), G$ is the CCD gain, $S$ the total counts from the star within the chosen aperture $A$ (in fractional pixels), and $\sigma_{\mathrm{B}}$ is the standard deviation of the background over the $N$ fractional pixels of a defined sky annulus around the main aperture. The first two terms inside the square root represent the error due to photon statistics from the star and the sky background respectively, while the third addendum accounts for the error due to subtraction of the mean sky.

The computation of the PSF photometric error involves several steps, as in this case the magnitude is calculated by an iterative least-square procedure (see Stetson 1987 for details). Briefly, the method consists in fitting the residuals of the pixel values after subtraction of the preliminary PSF profile; the standard error of the flux in each pixel is calculated from the readout noise and gain of the detector, and it is rigorously propagated through the least squares solution to predict the PSF magnitude error. After the fit has been perfomed, the rms value of the observed pixel residuals is computed. An average of these two estimates is returned as the final magnitude error, as implemented in the IRAF DAOPHOT package.

\subsection{Transformation to the Johnson-Kron-Cousins system}

Transformation of instrumental magnitudes onto the Johnson-Kron-Cousins system is achieved by imaging selected faint standard stars from the Landolt catalog. Exposures of the Landolt stars are taken several times during an observing night, with fairly large air mass distributions (typically $\sim 1.1-2$ ). Particular attention has been paid in culling the Landolt list, with the purpose of covering an adequate range of colors. Moreover, stars with a poor observation history, although not completely excluded, were seldom used. Finally, we used Landolt fields with more than one standard star in the CCD frame, thereby mitigating the negative effect of one poor observation on the final parameters of the fit. Landolt stars are visually identified in each exposure. Then, the objects in the $V$ and $B$ frames are linked to the corresponding ones in the $R$ frames. Finally, a fit of the observed to the standard magnitudes is performed using the IRAF task FITPARAM, and applying the following set of equations:

$\left\{\begin{array}{c}R=r+z_{R}+K_{R} \cdot X_{R}+\beta_{R} \cdot(V-R) \\ V=v+z_{V}+K_{V} \cdot X_{V}+\beta_{V} \cdot(B-V) \\ B=b+z_{B}+K_{B} \cdot X_{B}+\beta_{B} \cdot(B-V)\end{array}\right.$

where uppercase and lowercase letters indicate standard and instrumental magnitudes respectively, and $X_{R}, X_{V}, X_{B}$ are the air masses computed for each exposure. The magnitude scale zero-point is given by the $z$ terms, while the $K$ terms represent the first order extinction coefficients, and the $\beta$ terms gauge the color component of the transformation between the standard and the instrumental systems. As prescribed by Harris et al. (1981), a least-square solution of the above set of equations is carried out to estimate at once both the nightly atmospheric extinction values and the color transformation between the instrumental and the standard system. The actual value of the estimated parameters might vary from night to night, reflecting the statistical properties of the least-square solution. However, since the parameters are correlated with each other by the model, small errors 
of the extinction values are well compensated by adjustments in the color term estimates, making the results for the program stars fairly stable. An alternative expression for the $V$ equation, which have been implemented for part of the southern data reductions, makes use of the color $(V-R)$ instead of $(B-V)$, allowing one to account for a color term in the standardization of the $v$ magnitude where a $B$-filter exposure is not available. We verified that the $(B-V)$ and $(V-R)$ colors of the Landolt stars retained in our list are comparable in range and that they are linearly related; therefore, the photometric response in the $V$ band is equally well modelled by using either color. The task FITPARAM is used interactively, allowing visual inspection of the quality of the fit, and possibly reject dubious observations. If judged necessary, a temporal term of the form $\delta \times T_{\text {obs }}$ is added to the equations. This was seldom used to model constant temporal variations in the night extinction coefficients, or the presence of linear drifts of the detector/filter system. A typical rms of a good fit is of the order of $0.02-0.03$, whereas formal errors on the transformation coefficients are typically an order of magnitude smaller than their estimated values. When a satisfying fit could not be achieved the night was considered non photometric, and the newly observed program fields tagged for re-observation.

\subsection{Photometric errors in the standard system}

The final photometric error assigned to each object in a frame is computed by the IRAF task INVERFIT, with the default input option errors = "obserrors". This error estimate is an empirical one which takes into account the variance of the observation only, without propagating the errors of the coefficients of the transformation to the standard system. We have tested the reliability of this error estimate by direct comparison of independent observations of the same sequence. The results show that the current estimation is a lower limit for the expected photometric error. In particular, the uncertainty of the zero-point estimation (e.g., $z_{V}$ for the $V$ passband, but the same reasoning applies to all filters) adds to the final error budget a contribution comparable with that induced by the photon noise error of the stellar source, according to the approximate formula:

$\sigma_{V}^{2} \simeq\left(\frac{K_{B}+1}{K_{V}+1}\right)^{2} \sigma_{\text {obs }}^{2}+\left(\frac{K_{V}+1}{K_{B}+1}\right)^{2} \sigma_{z_{V}}^{2}$

Hence, while for stars fainter than $\sim 17$ the main error constribution is from photon noise, for brighter stars the zero-point uncertainty is typically higher than the instrumental magnitude one, and the IRAF-computed error is significantly underestimated. A more rigorous error propagation formula will be used for future GSPC-II releases.

\subsection{Astrometry}

Astrometry of each CCD frame is carried out as part of the reduction pipeline using the Digital Sky Survey (DSS,
Lasker 1994) as the reference catalog. The IRAF task employed is LYNXYMATCH (XYXYMATCH in the current IRAF version), which makes use of the triangularization algorithm (Groth 1986) to link reference to program stars in a completely automated fashion. If the algorithm fails, which occurred for about $25 \%$ of all cases, the user is asked to interactively identify the stars and restart the procedure. The astrometric accuracy of a GSPC-II object is limited by that of the DSS, which is typically of the order of half an arcsecond. On the other hand, their relative astrometric precision is generally better than the absolute one, typically $0.2-0.3$ arcsec and fairly homogeneous over the sky. It is to be noted however that for the $\sim 23$ arcmin CCD format the final astrometric error is somewhat critical, ultimately depending upon the distribution of the DSS stars found by the triangles algorithm on the CCD frame. More explicitely, if the stars found in the DSS cover a small area of the CCD frame, the error propagation of the parameters of the linear transformation could generate large errors in the extrapolated CCD area. Hence, one can expect a few cases where even a search aperture radius of 2-3 arcsec cannot ensure a good matching of GSPC-II with plate objects. We are in the process of correcting these astrometric errors as they become evident through the GSC-II plate processing pipeline.

\subsection{Applications to Schmidt photographic photometry}

Our experience with the calibration of Schmidt survey plates has demonstrated that an accurate response function to incident light can be obtained by fitting the logarithmic integrated density of stellar objects to some externally derived magnitudes, as long as their Point Spread Function (PSF) on the photographic emulsion is reasonably stable and they are not highly saturated; requirements which are clearly met by the Schmidt images of the stars in the GSPC-II sequences. However, GSPC-II standard magnitudes need to be converted to the passband defined by the plate emulsion/filter combination, before they can be used for plate calibration. Color transformations of the form $J=J(B-V, V), J=J(V-R, R)$ - where $J$ stands for the blue POSS-II passband - were derived for each of the GSC-II passbands, by using the STSDAS package SYNPHOT and a library of empirical and theoretical spectra (Lejeune et al. 1997; Gunn \& Stryker 1983). These transformations are strictly valid only within a particular color range $(-0.15<V-R<1.43)$, corresponding to mainly early- to medium-type main sequence stars. Similar constraints are enforced for the $B-V$ color.

Another crucial aspect of the photometric calibration deals with off-axis aberrations. These become noticeable at a radius of $\sim 2.7^{\circ}$ from the plate center and increases toward the edges, their main component being attributable to vignetting of the plate corrector, and therefore radially symmetric. Because of the non-linearity of the emulsion sensitivity, vignetting gives rise to a complex photometric response which needs to be properly calibrated by 
using photometric standards over a much larger plate area. To overcome this problem, we have recently secured special sets of calibration sequences, currently being used to build vignetting correction masks for each Schmidt survey/passband (García Yus 2000).

\section{Data-base organization}

Given the many sources of CCD and telescope data, as well as the time span of this project, our first goal toward a global error analysis was to develop a solid archiving system for comparing the results of independent photometric reductions and keeping on-line all the necessary information to perform an a posteriori data analysis. This was realized using the DIRA (Distributed Information Retrieval from Astronomical files, Merighi et al. 2000) database system. In summary, the pipeline generates two statistical tables per night: one containing frame object data, such as image features, $(x, y)$ coordinates, sky background, etc.; the other with the main features of each CCD exposure, e.g., seeing, average sky background, and number of objects detected in each frame. Finally, the main output file for a given night lists the star's standard photometry and astrometry, plus instrumental photometric data. These quantities are generated by the IRAF tasks PHOT and ALLSTAR, and stored in the form of IRAF tables. Subsequently, these tables are converted by the pipeline into DIRA-compatible ASCII files, which we call Intermediate Field Catalogs. An Intermediate Field Catalog (hereafter IFC) essentially contains $(B), V$, and $R$ magnitudes as obtained from the reduction of an ensemble of CCD frames intended to be a set of one-epoch, multi-color exposures for a particular GSPC-II field. Since our basic data quality check is the comparison between PSF and aperture photometry, a requisite for an object to be part of the IFC is that both its PSF- and aperturederived standard magnitudes be successfully computed. Each IFC record includes an object name (uniquely labelling the object within this sub-catalog), plus all the additional fields containing photometry, astrometry and other miscellaneous information.

\subsection{Object naming}

In the previous sub-section we have described how the DIRA database is populated with IFCs. At this point, we need to define some efficient criteria for incorporating the different standard magnitudes computed for a single stellar object belonging to different IFCs into a unique set of $B, V$, and $R$ magnitudes. Firstly, a directory-tree structure is created according to GSPC-II field names, with a single sub-directory assigned to each GSPC-II field. Secondly, each IFC file is stored in the sub-directory assigned to the proper GSPC-II field. Lastly, for each field, all the pre-selected files are inter-compared and the single objects matched against each other by using the IRAF task LINXYMATCH. The final names of the sequence objects are made up of a prefix identifying the corresponding survey plate, where the letters $\mathbf{N}, \mathbf{S}$ are used for the 1855 equinox northern grid and the 1950 equinox southern grid respectively, while $\mathbf{X}$ refers to the POSS-II survey grid. A 4-letter suffix ( $A A A A, A A A B, A A A C$, etc.) uniquely identifying the object within the sequence is appended to the name. At this stage, a field master list containing the matching status of individual objects in each IFC is produced. This master list includes the object names from the IFCs and their CCD frame positions transformed to a common reference system, which is realized by the $x, y$ coordinates of the $R$ frame of the field used to initiate the matching procedure. Moreover, to facilitate the retrieval of the relevant photometric and astrometric frame data, a pointer to the IFC record number is added. Any time a new IFC becomes avaliable, the matching task is run and the field master list is updated. Figure 2 summarizes the processing steps from CCD raw data to the matching of different IFCs for a generic GSPC-II field $k$. The next task is the validation and proper photometric average of the matched frame objects, in order to build an objectas opposed to a frame-catalog.

\section{The photometric sequences}

The experience acquired with the reduction and analysis of thousands of CCD frames from different observatories and telescopes led us to the conclusion that a thorough assessment of the photometric quality of each CCD object must be complemented by visual inspection of the frame data. On the other hand, the use of an automated pipeline has been essential to the project, by making feasible the re-reduction of a large part of the data with minimum effort, after some critical input values were established. We also verified that both PSF and aperture photometry carried out in a semi-automated way with the standard IRAF packages can have systematic errors, and a simple, yet effective rule to detect such errors is to check the two magnitudes against each other. Basically, we have adopted a set of tools for Quality Assurance (QA) of the sequences mainly serving the purpose of finding gross errors. Additionally, we have checked a number of common fields re-imaged at most observing runs in order to monitor the stability of the different equipment used and to ascertain all-sky data homogeneity.

\subsection{Selection criteria and caveats}

The criteria used for the selection of the sequences released with this paper are the following: a) sigma of unit weight of the fit to the Landolt stars to better than 0.05 mag; b) at least 6 stars per CCD sequence; c) single object instrumental error in each filter to better than 0.15 mag; d) agreement between PSF and aperture photometry.

The details of the implementation of criterion d) are explained in the following. For each frame a linear transformation between the PSF and aperture magnitudes is carried out according to the model $M_{\mathrm{apt}}-M_{\mathrm{psf}}=z_{0}+$ $k \cdot M_{\text {apt }}$. Then, a 3 -sigma rejection criterion is used to 


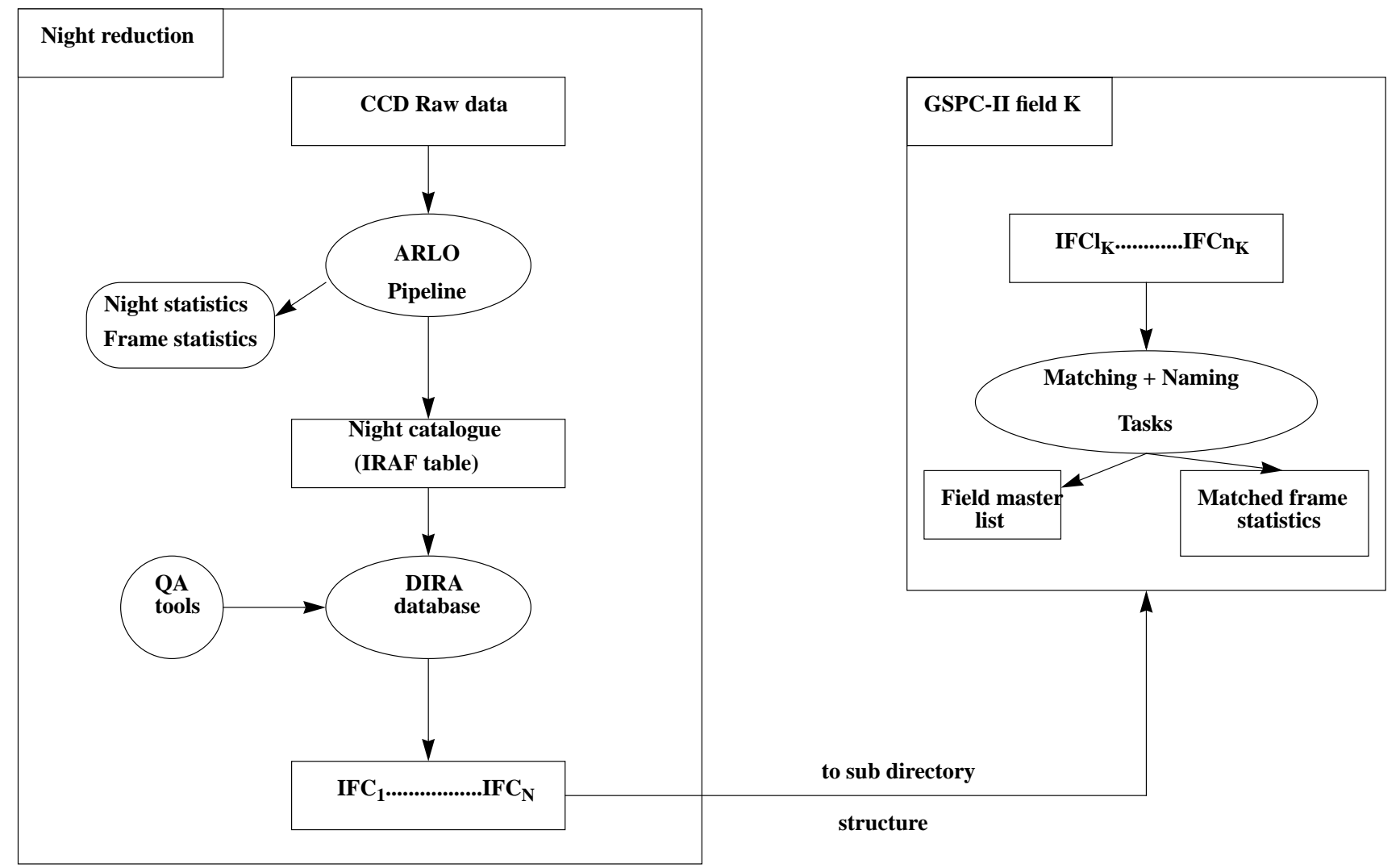

Fig. 2. Visualization of the main steps of the night-reduction pipeline (left), and of how the frame data is channeled into different GSPC-II field containers and matched against each other (right)

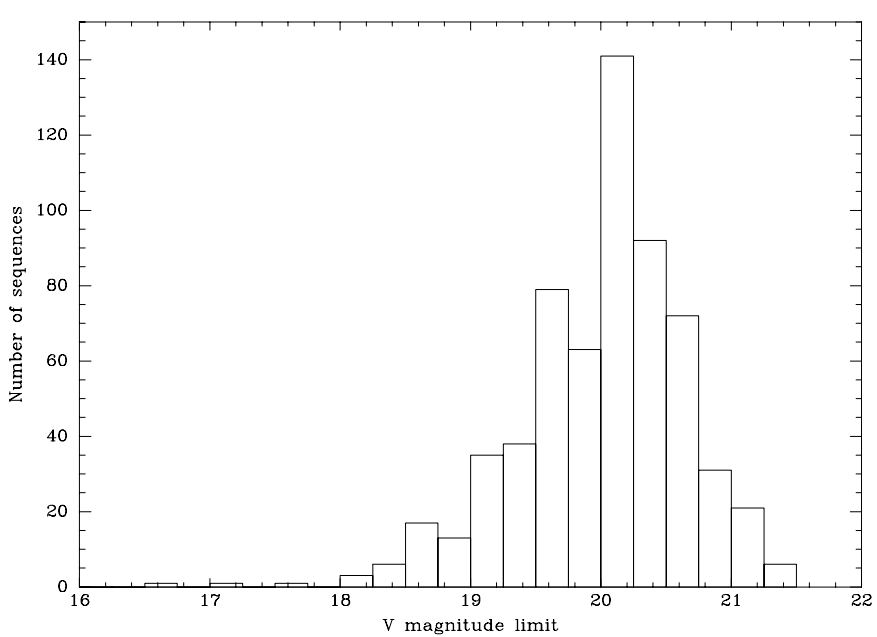

Fig. 3. The 622 GSPC-II fields, binned according to the limiting magnitude of the sequences

estimate the parameters of the fit, and their corresponding errors. Only sequences with $|k|<0.01$, and rms (sigma of unit weight $)<0.3$ in all filters are retained. These thresholds have been empirically determined, and have proven to be reliable for removing frames characterized by a poor choice of the PSF profile function. Once the frames have been selected, multiple observations (i.e., those coming from different IFCs) are averaged using equal weights to produce the best current estimate of the stellar
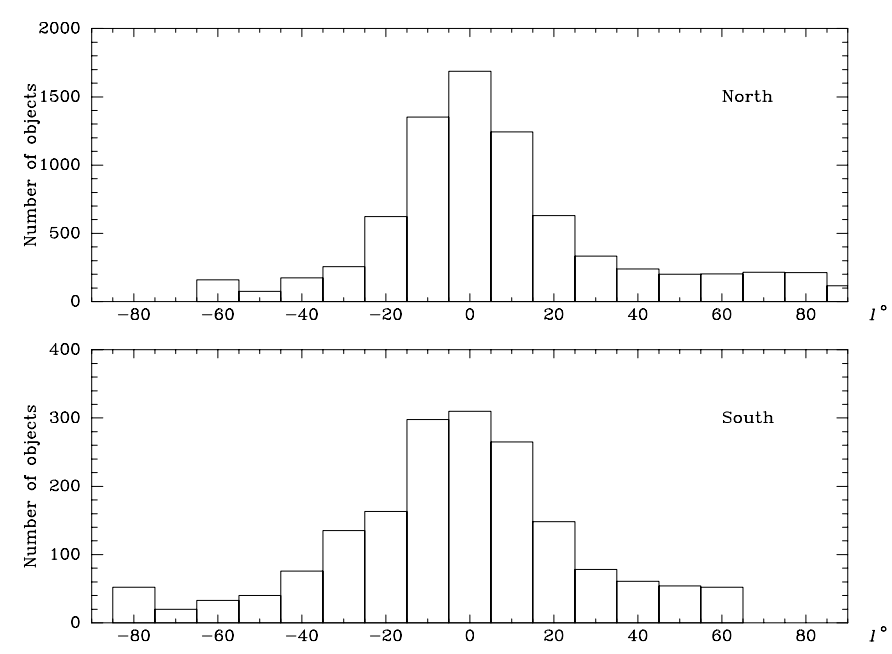

Fig. 4. Average number of GSPC-II objects per sequence as function of galactic latitude (upper and lower panel refer to Northern and Southern hemispheres respectively)

magnitudes. As already mentioned, these sequences are primarily intended for the calibration of Schmidt plate surveys. Therefore, they should be used as a whole to obtain a calibration curve. Accurate single object photometry can only be ensured by dedicating more time (in term of human resources) to the analysis of each single frame. 

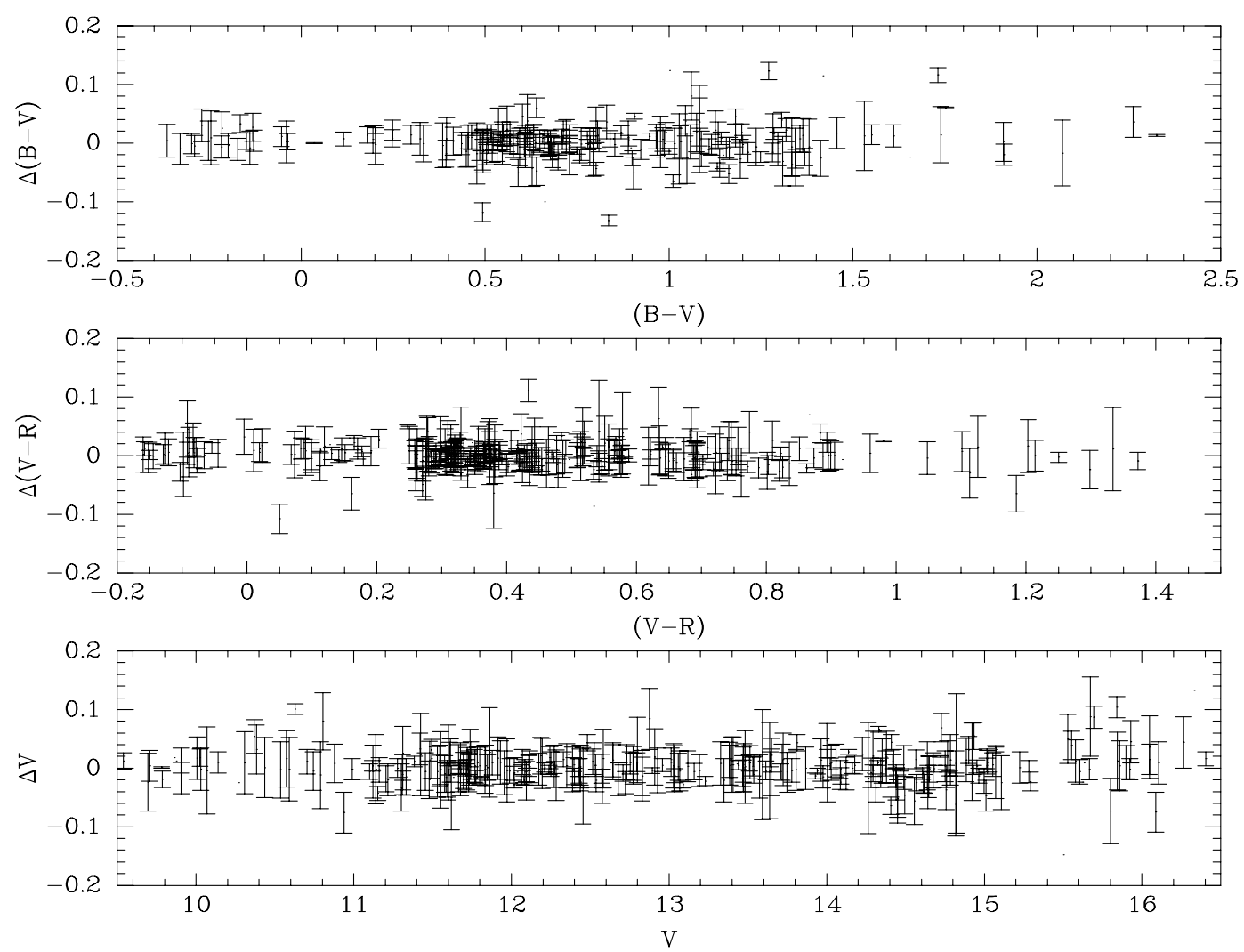

Fig. 5. Average differences between catalog and fitted magnitude and colors of the Landolt standard stars imaged at every GSPC-II observing run from the Kitt Peak, Cerro Tololo and ESO telescopes
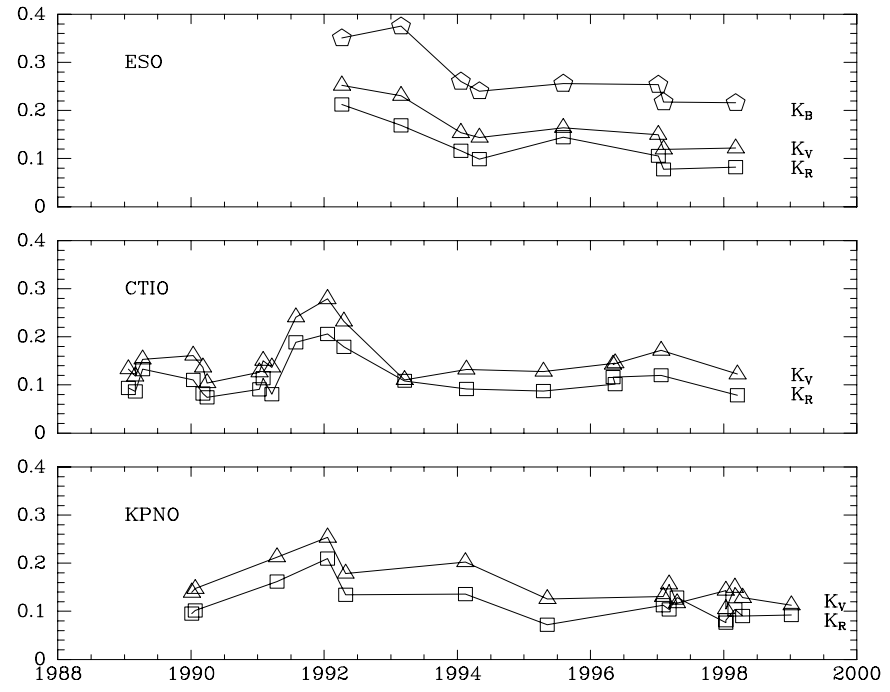

Fig. 6. Mean yearly extinction values in the $B, V$ and $R$ passbands for the Kitt Peak, Cerro Tololo and ESO-La Silla observatories

\subsection{Variability}

A related issue is the handling of candidate variable stars. As expected, our database will contain a percentage of variable stars with more than one observation over the years, which can be used to detect such variability. While the ultimate goal in such cases would be to distinguish between genuine variability and photometric errors of

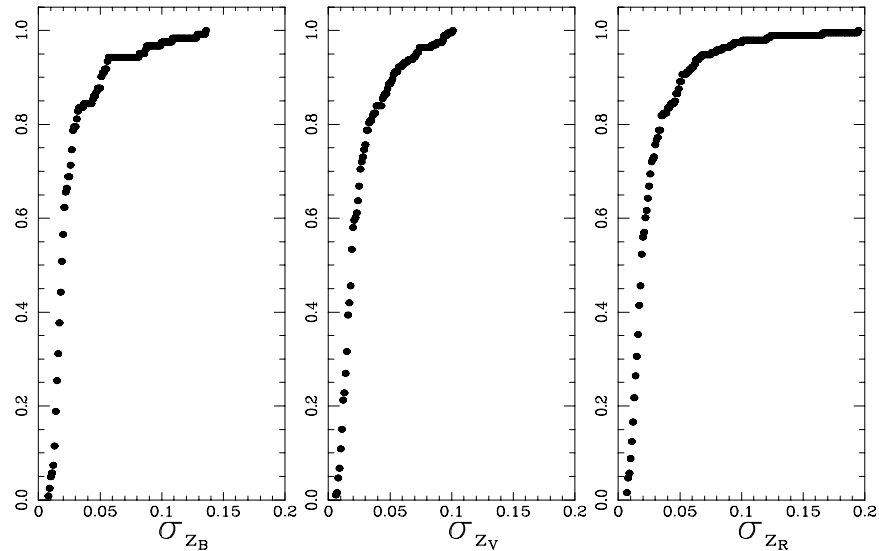

Fig. 7. Cumulative distributions (\%) of the zero-point error of the transformations to the standard system for the $B, V$, and $R$ passbands, from left to right respectively

various nature, variability is not taken into account in the current catalog release, and magnitudes coming from different frames are simply averaged to obtain the final photometry.

\subsection{Global statistics}

The results reported here refer to the photometric sequences selected with the criteria described in the previous subsections. Data are from 7 different telescopes (see 
Table 1; data from Mt. Hopkins, Lowell, and McDonald Observatories were not included in this release), for a total of 153 observing nights, 1285 IFCs and 622 different GSPC-II fields. The open circles of Fig. 1 show the equatorial position of the centers of the selected fields. The total number of catalog objects, after averaging multiple observations (see Sect. 5.1), is 219 942. The histogram of Fig. 3 represents the number of sequences as function of limiting magnitude in the $V$ passband. The distribution is well peaked around $V_{\text {lim }} \simeq 20$. The 3 bright sequences $\left(V_{\lim }<18\right)$ correspond to southern fields S171, S226 and S311 for which only short exposures are available in the present catalog version. The average number of objects per sequence is displayed in Fig. 4 as function of galactic latitude. It can be noted that the northern sequences are on average more populated than the southern ones, due to the larger CCD formats used.

In Fig. 5 the mean differences between the Landolt catalog values and the ones estimated by the nightly fits of the observations are plotted as function of color for the complete set of Landolt stars used to reduce our data. For each star, an empirical estimation of the error on the fitted Landolt photometry is obtained by summing the differences in quadrature, and the resulting error bars are plotted. As the figures show, the general agreement of the Landolt catalog stars with their fitted values is within few percent, and there is no evidence of residual systematic color or magnitude effects at this level of accuracy.

As the observations have been carried out over several years, we looked for possible long-term trends in the value of the extinction coefficients. Average estimates of these values as a function of time are reported in Fig. 6 for the Kitt Peak, Cerro Tololo, and ESO-La Silla telescopes, for which the data statistics are more significant. It is interesting to note that the extinction (in all filters) show the effects of the 1991 eruption from the volcano Pinatubo in the Philippines.

As explained in Sect. 4.3, an important contribution to the final photometric error comes from the zero-point error of the transformation between instrumental and standard photometry. In Fig. 7 the cumulative distribution of the zero-point errors, as estimated by the IRAF task FITPARAM, is shown for all the selected nights. The percentage of nights with zero-point errors smaller than 0.06 is $\sim 95 \%$.

\subsection{Photometric accuracy}

The first independent check of the photometry quality of our data has been obtained by comparison with the faint GSPC-I stars, corresponding to the bright end of the GSPC-II sequences. As GSPC-I objects appear saturated in our long CCD exposures, we have pre-selected 5 and 4 min exposures (or shorter) in the $B$ and $V$ band respectively before matching GSPC-II objects with GSPC-I. The match resulted in 597 objects in the $V$ passband and 380 objects in $B$. From this comparison we detected a few
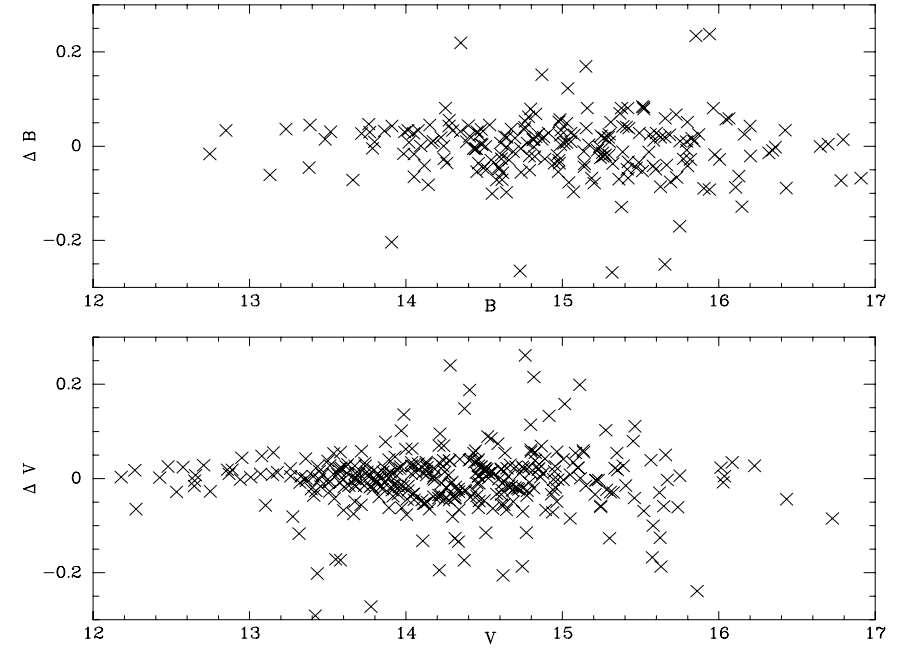

Fig. 8. GSPC-I/GSPC-II $B$ magnitude (top panel) and $V$ magnitude (bottom panel) residual plot

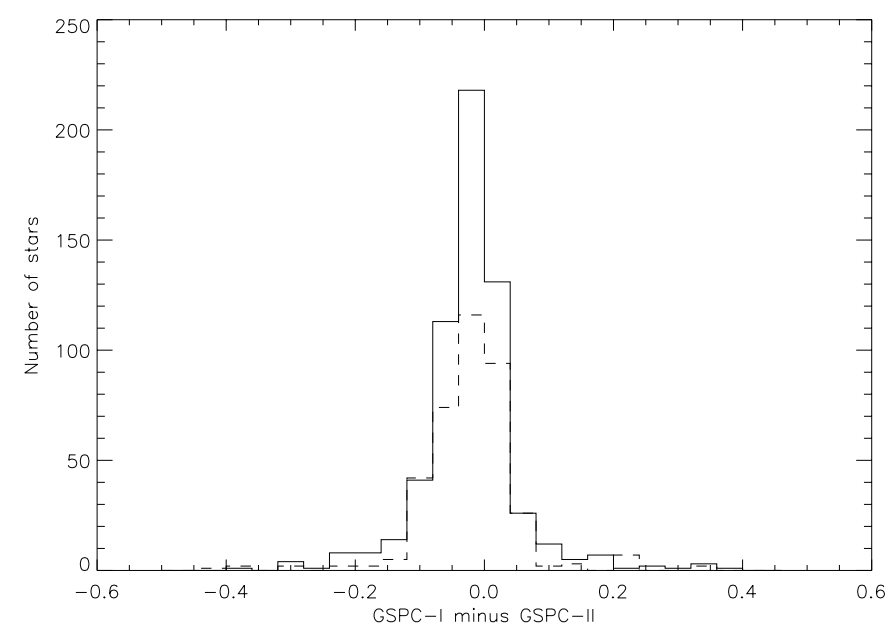

Fig. 9. Distribution of GSPC-I/GSPC-II magnitude differences ( $V$ mag: solid line; $B$ mag: dashed line)

outliers, some of which could be explained by poor observation history of the GSPC-I stars; globally, the agreement between GSPC-II and GSPC-I photometry is at the level of few percent, as the residual plots and histograms of Figs. 8 and 9 indicate. The statistics computed on these residuals, without eliminating any outlier, give an rms of $0.07 \mathrm{mag}$ and a mean of 1 millimag in the $V$ passband, while the analogue values for the $B$ filter are 0.06 mag and 3 millimag.

To test the average photometric quality of the standard sequences down to the magnitude limit, we have intercompared observations of the same GSPC-II fields coming from different runs at the main telescopes used for this program. After the selection criteria delineated in Sect. 5.1 had been applied, we found 46 GSPC-II fields in common between the CTIO and ESO-La Silla observatories, for a total of 150 different IFCs (see Sect. 4), and 10 GSPC-II fields - only one common field in the $B$ filter - shared between KPNO and CTIO, corresponding to 33 IFCs. The magnitude differences of all objects 

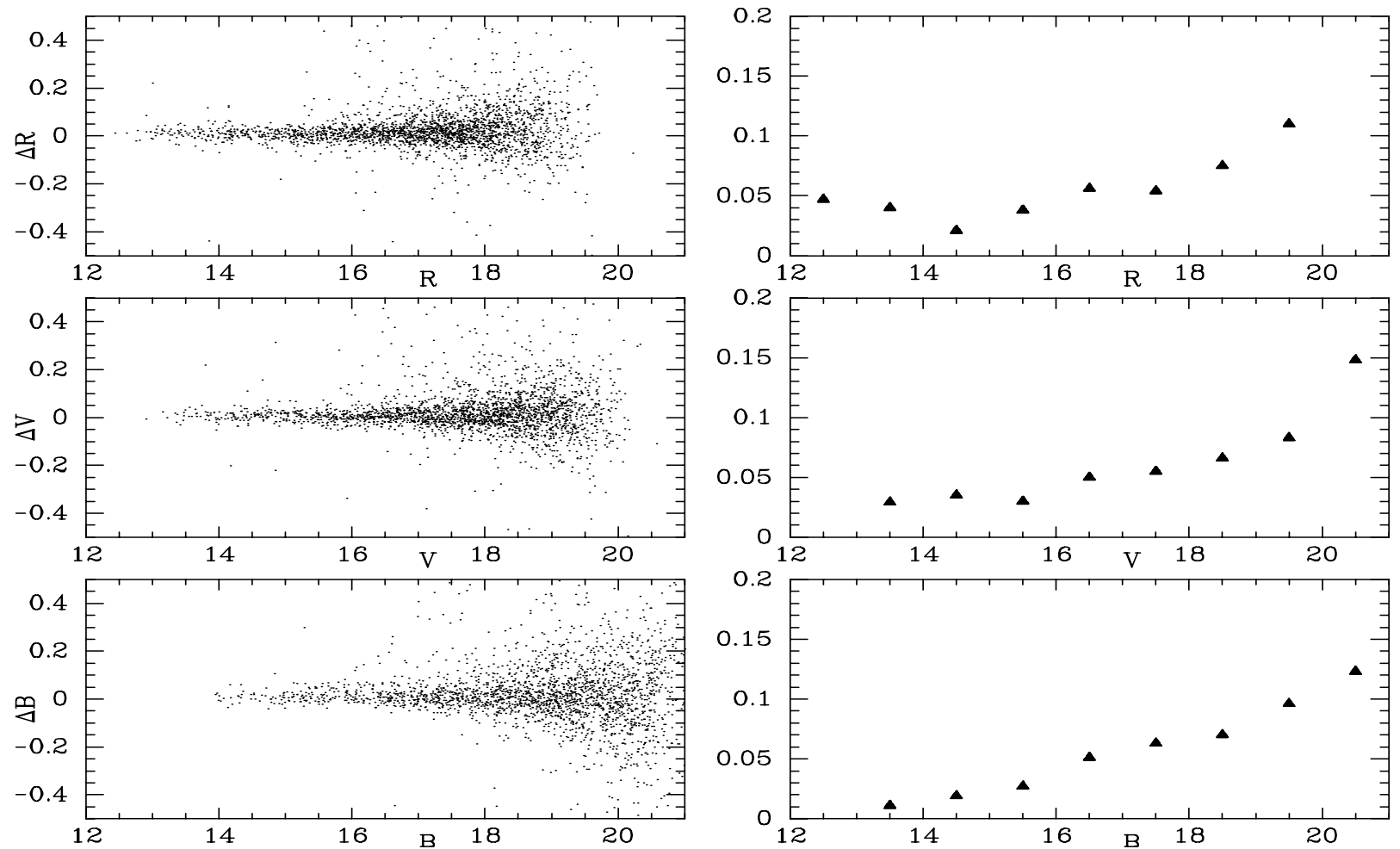

Fig. 10. Comparison of 46 common fields between CTIO and ESO. Each point in left panels represents the difference between two standard magnitudes of the same star as measured independently at CTIO and ESO, in the $B, V$, and $R$ passbands. The graphs on the right show the corresponding $1-\sigma$ photometric error per magnitude bin, as measured from the dispersion of the points in the corresponding the left panel
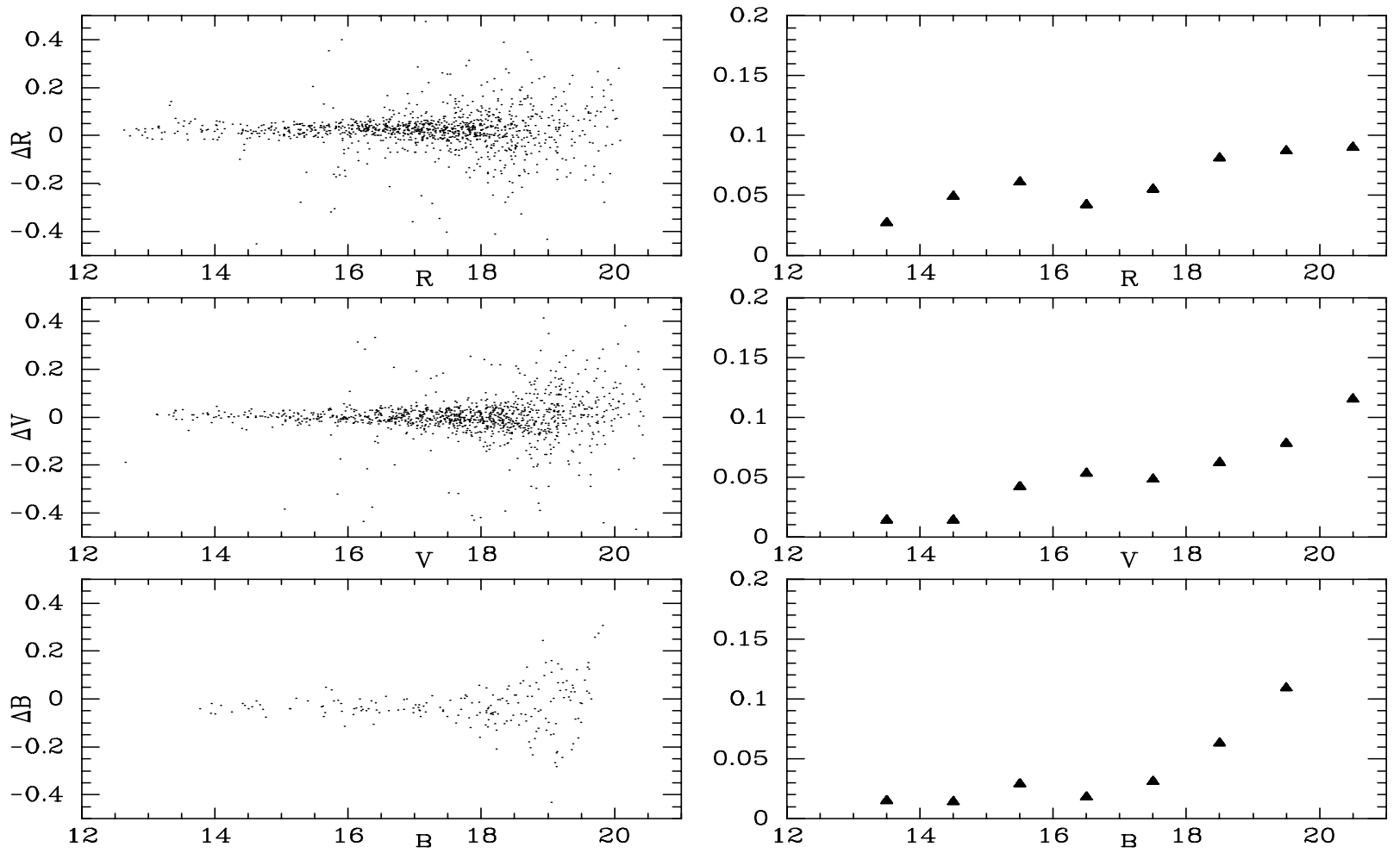

Fig. 11. Comparison of 10 common GSPC-II fields between CTIO and Kitt Peak observatories in the $V$ and $R$ filters. Only one common field (N507) was available in the $B$ passband. See explanatory caption of Fig. 10 
in common between different IFCs are plotted in Figs. 10 and 11 for each passband and for the two inter-observatory comparisons. Then, by binning the stars into appropriate magnitude ranges we estimated the single-object photometric error as a function of magnitude, which is reported in the right panels.

The graphs show fluctuations of the estimated error vs. magnitude which reflect the presence of some outliers, although the general behaviour of the curves is fairly stable, with an error at 19 mag of approximately $0.07 \mathrm{mag}$. Possible causes of these outliers, which are not identifiable with any particular field or CCD frame, are: object misidentification, crowded-field effects, stellar variability. It is conceivable that, by devoting more time to the quality control of the data, a large part of such outliers can be detected and removed from the sequences. As some of them will go undetected when we have only one GSPC-II frame, it is important to note that GSC-II reductions have a considerable robustness to them because there typically are many faint stars in each sequence - and in particular in the crowded ones - so that the outliers will have large residuals in the photographic photometry and will therefore be rejected. As a last comment, photometry which suffered from light contamination by a close object(s) on the CCD frame is usually excluded from the calibration process, since those stars would appear as blends in the Schmidt photographic plate. Such cases will be detected and removed by comparison of aperture and PSF photometry in future catalog releases.

\subsection{The export catalog}

As outlined in Sect. 5.1, multiple observations of the same GSPC-II stars have been averaged, and the mean value is taken to be the best estimation of the object's magnitude. Each catalog record contains an object identifier, according to the GSPC-II nomenclature given in Sect. 4.1, its equatorial coordinates at equinox J2000, plus its magnitude and magnitude error in the $B, V$, and $R$ bands respectively. When the $B$ magnitude information is not present, the corresponding fields are left blank. The reported magnitude error (in each passband) is obtained by the formal error propagation of individual standard errors coming from the reduction of different sets of exposures pertaining to that object, as computed by IRAF. Since, as pointed out in Sect. 3.3, the standard error computed by IRAF can be an underestimate of the true one, we also computed an empirical $\sigma_{\text {mag }}$ when more than one IFC was available. Objects for which the empirical photometric error exceed $0.25 \mathrm{mag}$, and which represented only $1 \%$ of the data set selected by the criteria exposed in Sect. 5.1, did not enter the export catalog.

\section{Conclusions and future work}

The 622 photometric sequences released with this paper are made available through $\mathrm{ftp}$ at both STScI (www.stsci.edu) and OATo (www.to.astro.it) web sites. These sequences represent about one third of the total number of GSPC-II target fields. Our present efforts are focusing on quality control of the remainder of the in-house data, and on (re-)observations of the missing sequences, with the primary goal of completing the sky coverage in the $(B), V$, and $R$ passbands.

As resources permit, we also plan to improve the photometric quality of the sequences by using PSF - as opposed to aperture - photometry. This will require the rereduction of all the CCD frames where the automated choice of the PSF stars failed to give good results.

In addition to that, we are considering the possibility of completing the observations in the $B$ passband and are also evaluating the costs/returns of adding an I passband to the sequences, which would significantly improve the calibration of the near-IR Schmidt plates. Given that the realization of such a project in a foreseable time-span would yield a strong impact on the use of photographic sky surveys for new-generation telescope operations, space missions and for astrophysical investigations, its feasibility will ultimately be subject to resources and funding available at both institutions.

Acknowledgements. The long-term endorsment by the ESO-La Silla and NOAO CTIO and KPNO Observatories time allocation panels has made the accomplishment of this effort a reality.

We wish to thank R. Giacconi, A. Ferrari, and P. Benvenuti for their constant support toward the success of this international collaboration.

The GSPC-II project has been partially funded by ESO/ST-ECF under contracts 56815/ECF/99/6302/NKA and 56186/ECF/99/6303/NKA.

Space Telescope Science Institute is operated by the Association of Universities for Research in Astronomy, for the National Areonautics and Space Administration under contract NAS5-26555.

\section{References}

Bucciarelli, B. 1999, The GSC-II Project: Status and Overview, in Treasure Hunting in Astronomical Plate Archives International Workshop, ed. P. P. Kroll, et al. (Verlag Harri Deutsch), 45

Cannon, R. D. 1984, Sky Surveys with the UK 12 m Schmidt Telescope, in Astronomy with Schmidt-Type Telescopes, ed. M. Capaccioli (Reidel, Dordrecht), 25

Casalegno, R. 1998, OATo Internal Report, No. 44

Casalegno, R., Bucciarelli, B., García, J., \& Lasker, B. M. 1999, ASP Conf. Ser., 189, 154

García Yus, J. 2000, Internal Report

Groth, E. J. 1986, AJ, 91, 1244

Gunn, J. E., \& Stryker, L. L. 1983, ApJ, 52, 121

Harris, W. E., Fitzgerald, \& Reed, B. C. 1981, PASP, 93, 507

Landolt, A. U. 1992, AJ, 104, 340

Lasker, B. M., Sturch, C. R., Lopez, C., et al. 1988, ApJS, 68, 1

Lasker, B. M., Sturch, C. R., McLean, B. J., et al. 1990, AJ, 99, 2019

Lasker, B. M. 1994, Digitization Programs at STScI, in Astronomy from wide-field imaging: proceedings of the 161st IAU Symp., ed. H. T. MacGillivray, et al. (Kluwer Academic Publishers, Dordrecht), 167 
Lasker, B. M., McLean, B. J., Jenkner, H., Lattanzi, M. G., \& Spagna, A. 1995, in Future Possibility for Astrometry in Space, ESA SP-379, 137

Lejeune, Th., Cuisinier, F., \& Buser, R. 1997, A\&AS, 125, 229

Massey, P., Garmany, C. D., Silkey, M., \& Degioia-Eastwood, K. 1989, AJ, 97, 107

McLean, B., Greene, G., Lattanzi, M., et al. 2000, An Overview of the Multi-Terabyte GSC-II Database and some Preliminary Scientific Resluts, in MPA/ESO/MPE Joint Astronomy conference "Mining the Sky" Garching (Germany), July 31 - August 4 (Springer-Verlag) in press

Merighi, R., Morbidelli, R., Nanni, M., \& Tinarelli, F. 2000, D.I.R.A.2 User Manual, Astronet Doc. Service, adoc@astrts.astro.it

Minkowski, R. L. \& Abell, G. O. 1963, in Basic Astronomical Data, ed. K. A. Strand (Univ. of Chicago Press, Chicago), 481
Morgan, D. H., Tritton, S. B., Savage, A., Hartley, M., \& Cannon, R. D. 1992, Current and Future Programmes with the UK Schmidt Telescope, in Proceedings of the Conference on "Digitised Optical Sky Surveys", ed. H. T. MacGillivray, \& E. B. Thompson (Kluwer Academic Pub., Dordrecht), 11

Reid, I. N., Brewer, C., Brucato, R. J., et al. 1991, PASP, 103, 661

Sciortino, S., Micela, G., Favata, F., Spagna, A., Lattanzi, M. G. 2000, A\&A, 357, 460

Spagna, A., Lattanzi, M. G., Lasker, B. M., et al. 1996, A\&A, 311,758

Spagna, A., Lattanzi, M., McLean, Massone, G., \& Lasker, B. M. 1998, A\&AS, 130, 359

Stetson, P. B. 1987, PASP, 99, 223

Terranegra, L., Morale, F., Spagna, A., Massone, G., Lattanzi, M. G. 1999, A\&A, 341, L79 